\title{
Efeitos do ultra-som terapêutico contínuo sobre a proliferação e viabilidade de células musculares $\mathrm{C} 2 \mathrm{Cl} 2$ \author{
of C2C12 muscle cells
} \\ Effects of continuous therapeutic ultrasound on proliferation and viability
}

\author{
Paola Pelegrineli Artilheiro, Elisangela Nascimento Oliveira ${ }^{2}$, Crislene Sampaio Viscardi ${ }^{1}$, Manoela Domingues Martins, \\ Sandra Kalil Bussadori ${ }^{3}$, Kristianne Porta Santos Fernandes ${ }^{3}$, Raquel Agnelli Mesquita-Ferrari ${ }^{3}$
}

Estudo desenvolvido no Mestrado em Ciências da Reabilitação, Laboratório de Pesquisa da Unidade Vergueiro da Uninove - Universidade Nove de Julho, São Paulo, SP, Brasil

1 Mestrandas no Programa de Mestrado em Ciências da Reabilitação da Uninove

2 Fisioterapeuta Ms.

3 Profas. Dras do Programa de Mestrado em Ciências da Reabilitação da Uninove

\section{ENDEREÇO PARA \\ CORRESPONDÊNCIA}

Raquel A. Mesquita-Ferrari Pós-Graduação em Ciências da Reabilitação / Uninove

Avv. Francisco Matarazzo 612

Água Branca

05001-100 São Paulo SP e-mail:

raquel.mesquita@gmail.com

O estudo recebeu financiamento da Fapesp - Fundação de Amparo à Pesquisa do Estado de São Paulo - e da Uninove.

APRESENTAÇÃo dez. 2009

ACEITO PARA PUBLICAÇÃO abr. 2010
Resumo: O ultra-som terapêutico (US) é um recurso bioestimulante utilizado para propiciar reparo muscular de melhor qualidade e menor duração, mas o potencial terapêutico do US contínuo não está totalmente estabelecido. O objetivo deste trabalho foi avaliar o efeito do US contínuo sobre a proliferação e viabilidade de células musculares precursoras (mioblastos C2C12). Mioblastos C2C12 foram cultivados em meio de cultura contendo $10 \%$ de soro fetal bovino e irradiados com US contínuo nas freqüências de 1 e $3 \mathrm{MHz}$ nas intensidades de 0,2 e 0,5 W/ $\mathrm{cm} 2$, durante 2 e 5 minutos. A viabilidade e proliferação celular foram avaliadas após 24, 48 e 72 h de incubação. Grupos não-irradiados serviram como controle. Foram realizados experimentos independentes em cada condição acima, e os dados obtidos submetidos à análise estatística. Os resultados mostram que não houve diferença estatisticamente significativa na proliferação e viabilidade celular entre os mioblastos tratados com US e as culturas controles após os diferentes períodos de incubação, em todos os parâmetros avaliados. Conclui-se que o US contínuo, nos parâmetros avaliados, não foi capaz de alterar a proliferação e viabilidade dos mioblastos.

DescritoRes: Células musculares; Mioblastos; Proliferação de células; Terapia por ultrassom

ABSTRACT: Therapeutic ultrasound (US) is a biophysical stimulation resource widely used in order to promote better, faster muscle repair, but the effectiveness of continuous US in treating injuries is not fully established. The aim of the present in vitro study was to assess the effects of continuous ultrasound on viability and proliferation of skeletal muscle precursor cells (C2C12 myoblasts). C2C12 myoblasts were cultured in a medium containing $10 \%$ foetal bovine serum and irradiated with continuous ultrasound at 1 and $3 \mathrm{MHz}$ frequencies, at intensities of 0.2 and $0.5 \mathrm{~W} / \mathrm{cm}^{2}$ for 2 and 5 minutes. Cell viability and proliferation were assessed after different incubation periods (24, 48 and 72 h). Non-irradiated groups served as control and data were statistically analysed. Results showed that no significant differences in cell viability or proliferation could be found between ultrasoundtreated myoblasts and control cultures under all test parameters and durations. Hence continuous ultrasound, at the used parameters, was unable to alter myoblast proliferation and viability.

KeY words: Cell proliferation; Muscle cells/ultrasonography; Myoblasts; Ultrasonic therapy 


\section{INTRODUÇÃO}

O músculo esquelético é um tecido dinâmico com alta capacidade de adaptação frente a estímulos fisiológicos como, por exemplo, treinamento físico ou lesões severas. As lesões musculares são muito freqüentes na prática desportiva, podendo ocorrer por diversos mecanismos, abrangendo desde trauma direto como lacerações e contusões, até trauma indireto como distensão, isquemia e disfunção neurológica ${ }^{1,2}$.

A formação do músculo esquelético ou miogênese é um processo complexo que envolve a expansão de células musculares mononucleadas progenitoras ao longo da via miogênica até se tornarem mioblastos que se fundem para formar miotubos e que, finalmente, se desenvolvem para se tornar miofibrilas do músculo esquelético maduro ${ }^{3,4}$. Quando um músculo sofre uma lesão, tem a habilidade de iniciar um processo de reparo altamente organizado de forma a prevenir a perda de massa muscular. Esse processo é semelhante à miogênese, porém as células que participam desse processo são as células satélites ${ }^{2,5}$.

Há grande interesse no estabelecimento de recursos e terapias a serem utilizados para tentar proporcionar um processo de reparo muscular de melhor qualidade e menor duração. O US terapêutico (US) é um desses recursos, que vem sendo cada vez mais utilizado para o tratamento de condições clínicas como lesões ligamentares, tendinites e lesões tendinosas, lacerações ou outros tipos de lesão tecidual, cicatrizes, úlceras varicosas, neuromas, processos inflamatórios articulares, fasceítes e, mais recentemente, no reparo de fraturas ósseas ${ }^{6,7}$, lesões musculares e trombose ${ }^{8-11}$.

Segundo Mendonça et al. ${ }^{12}$, há um consenso de que o US promove efeito benéfico sobre diversos tecidos, destacando-se dentre outros o aumento da angiogênese, do tecido de granulação, do número de fibroblastos e da síntese de colágeno, e a diminuição de leucócitos e macrófagos, nos quais já foi demonstrado o aumento da velocidade de cicatrização, a diminuição do número de células inflamatórias e a melhora da qualidade do tecido neoformado. Entretanto, ainda são escassas, e por vezes contraditórias, as evidências científicas que determinem com segurança os parâmetros dosimétricos e metodológicos necessários à aquisição desses objetivos por esse recurso ${ }^{11,13-18}$.

Estudos in vitro demonstraram que os efeitos do US parecem depender do tipo celular: promoveriam a síntese de DNA em osteoblastos humanos, fibroblastos de gengiva e pele, células de periósteo, mas não em condrócitos6. Quanto ao efeito do US sobre a proliferação celular, foi verificado inicialmente por Johns et al. ${ }^{19}$ que o US contínuo e pulsado, em intensidades de 0,1 a $0,7 \mathrm{~W} / \mathrm{cm}^{2}$, foi capaz de diminuir a taxa de crescimento celular; porém, em estudos posteriores, evidenciou-se que o US nas freqüências de 1 e $3 \mathrm{MHz}$ em intensidades variadas promovia aumento da proliferação de fibroblastos e osteoblastos ${ }^{20-22}$. Os estudos mais recentes que avaliaram o potencial terapêutico do US foram realizados em osteoblastos $^{23,24}$, condrócitos ${ }^{25-27}$, fibroblas$\operatorname{tos}^{28}$, células epiteliais ${ }^{29}$ e células endoteliais ${ }^{30}$. Um estudo prévio (não publicado) destes autores evidenciou que o US pulsado não interferiu na proliferação e viabilidade de células musculares.

O objetivo deste estudo foi verificar os efeitos do US contínuo nas freqüências de 1 e 3 Mhz, nas intensidades de 0,2 e $0,5 \mathrm{~W} / \mathrm{cm}^{2}$, durante 2 e 5 minutos, sobre a viabilidade e proliferação de mioblastos após diferentes períodos de incubação (24, 48 e 72 h). Com esse propósito, foram utilizados mioblastos $\mathrm{C} 2 \mathrm{C} 12$ que derivam de músculo esquelético de camundongos e exibem a maioria das características dos mioblastos normais (diferenciam-se em cultura), propiciando um bom modelo para estudar a regeneração muscular ${ }^{3,31-33}$

\section{METODOLOGIA}

A linhagem celular C2C12 aqui utilizada é um subclone da linhagem celular de mioblastos $\mathrm{C} 2$, células derivadas de células-satélite de ratos adultos ${ }^{28,29}$. Essas células foram cultivadas no meio de cultura de Eagle modificado por Dulbecco (DMEM, Cultilab, Campinas, SP, BR) contendo $10 \%$ de soro fetal bovino (SFB - Cultilab) e 1\% antibiótico solução antimicótica (Cultilab) e mantidas em estufa $37^{\circ} \mathrm{C}$, numa atmosfera úmida contendo $5 \%$ de $\mathrm{CO}^{2}$. O monitoramento do crescimento celular foi feito a cada 24 horas e, quando a monocamada celular se tornava subconfluente para a perpetuação da linhagem celular, foi realizado o subcultivo com lavagem tampão PBS1X ( $\mathrm{NaCl} 140$ mM; KCl 2,5 mM; $\mathrm{Na}_{2} \mathrm{HPO}_{4} 8 \mathrm{mM} ; \mathrm{KH}_{2} \mathrm{PO} 1,4$ $\mathrm{mM} ; \mathrm{pH} 7,4)$ e solução de tripsina. As células foram centrifugadas a 1200 rpm e posteriormente ressuspensas em $1 \mathrm{ml}$ de meio DMEM. A viabilidade das células foi avaliada por contagem com corante vital azul de trypan (0,4\%) e foram utilizadas nos experimentos as células com viabilidade maior que 95\%.

\section{Procedimentos}

O equipamento de US utilizado foi o Sonomaster Microcontrolled Comunicator (KW, Amparo, SP, BR) e os parâmetros de tratamento selecionados foram as intensidades de 0,2 e 0,5 W/cm², freqüências de 1 e $3 \mathrm{MHz}$ e modo contínuo de aplicação. A irradiação com US foi realizada conforme o modelo proposto por Mukai et al. ${ }^{27}$ : as células, suspensas em meio de cultura num tubo cônico estéril de $50 \mathrm{ml}$ foram centrifugadas a 1200 rpm (Centrífuga Excelsa ${ }^{4}$ modelo 280R - Fanem, São Paulo, SP, BR) para obtenção do precipitado celular. Em seguida, esse tubo foi submerso em água deionizada em temperatura de $37^{\circ} \mathrm{C}$, mantida constante com o auxílio de Banho-maria (Nova Técnica NT271, Piracicaba, SP, BR). A cabeça do transdutor foi posicionada perpendicularmente ao tubo de cultivo celular e fixada na armação, permanecendo a $3 \mathrm{~cm}$ da cultura celular. O tanque de água com dimensão interna de $16 \times 30 \times 49 \mathrm{~cm}$ foi revestido por placas de borracha com o intuito de eliminar reflexos múltiplos (Figura 1). O grupo controle sofreu a mesma manipulação, mas as células não foram irradiadas. A técnica subaquática foi utilizada, pois a água destilada ou desgaseificada permite melhor transferência da onda ultra-sônica, em termos de propriedade de impedância acústica ${ }^{27}$.

As células foram divididas em nove grupos experimentais, sendo um controle (sem tratamento) e oito grupos tratados com o US utilizando diferentes parâmetros, como sintetizado no Quadro 1. 


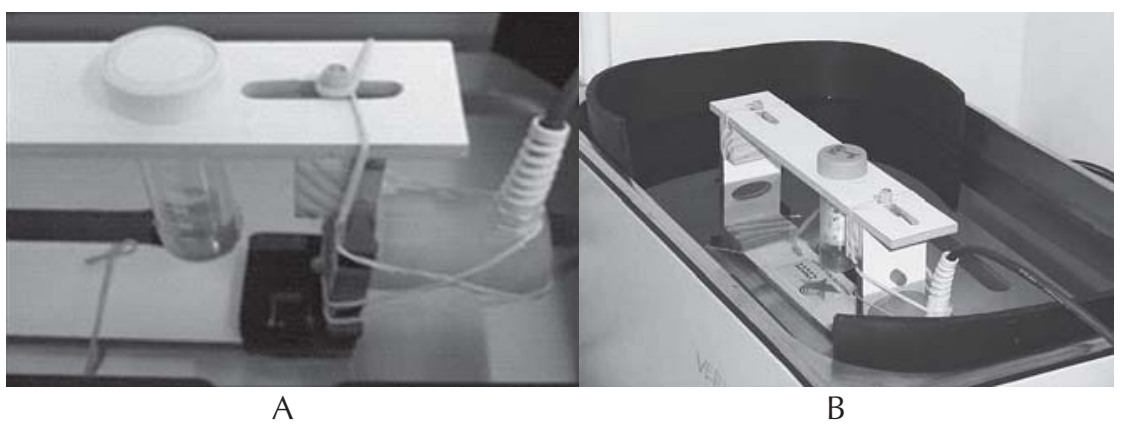

Figura 1 Ilustração: equipamento para irradiação dos mioblastos com US

(A) Aparato confeccionado com base no modelo proposto por Mukai et al. ${ }^{31}$

(B) Irradiação das células por US de forma subaquática

Quadro 1 Grupos experimentais e parâmetros dosimétricos usados para a irradiação com US

\begin{tabular}{|c|c|c|c|}
\hline Grupo & $\begin{array}{l}\text { Freqüência } \\
(\mathrm{MHz})\end{array}$ & $\begin{array}{l}\text { T irradiação } \\
\text { (minutos) }\end{array}$ & $\begin{array}{l}\text { Intensidd } \\
\left(\mathrm{W} / \mathrm{cm}^{2}\right)\end{array}$ \\
\hline $1 \mathrm{C}$ & - & Só imersão & - \\
\hline 2 & 1 & 2 & 0,2 \\
\hline 3 & 1 & 2 & 0,5 \\
\hline 4 & 1 & 5 & 0,2 \\
\hline 5 & 1 & 5 & 0,5 \\
\hline 6 & 3 & 2 & 0,2 \\
\hline 7 & 3 & 2 & 0,5 \\
\hline 8 & 3 & 5 & 0,2 \\
\hline 9 & 3 & 5 & 0,5 \\
\hline
\end{tabular}

$\mathrm{C}=$ Controle; $\mathrm{T}=$ tempo; Intensidd $=$ intensidade

A viabilidade (proliferação) celular foi avaliada após 24, 48 e 72 h do procedimento de irradiação com US. A metodologia utilizada baseia-se na habilidade da enzima mitocondrial desidrogenase, encontrada somente em células viáveis, em clivar os anéis de tetrazólio do MTT - 3-[4,5-dimethylthiazol-2yl]-2,5diphenyltetrazolium bromide; thiazolyl blue (Sigma-Aldrich, St. Louis, MO, USA) - formando cristais azul-escuros de formazana, que são impermeáveis às membranas celulares, ficando então retidos no interior das células viáveis ${ }^{34}$. A posterior lise celular faz com que esses sais de formazana sejam liberados. O número de células viáveis é diretamente proporcional ao nível de cristais de azul de formazana formados.

Após a irradiação com US, 1×103 células/poço, mantidas em DMEM con- tendo $10 \%$ SFB, foram adicionadas a placas de cultura de fundo chato de 96 poços estéreis (Costar) e incubadas a $37^{\circ} \mathrm{C}$ e $5 \% \mathrm{CO}_{2}$ durante os diferentes períodos avaliados, ou seja, 24, 48 e 72 horas. Ao término do período de incubação, foi feita a lavagem dos poços com PBS1X ( $\mathrm{NaCl} 140 \mathrm{mM}$; $\mathrm{KCl} 2,5 \mathrm{mM}$; $\mathrm{Na}_{2} \mathrm{HPO}_{4} 8 \mathrm{mM} ; \mathrm{KH}_{2} \mathrm{PO} 1,4 \mathrm{mM} ; \mathrm{pH}$ $7,4)$ para remoção das células mortas e adicionado o MTT $(0,5 \mu \mathrm{g} / \mathrm{ml})$; as células foram então incubadas por $4 \mathrm{~h}$ a $37^{\circ} \mathrm{C}$ e $5 \% \mathrm{CO}_{2}$. Em seguida, foi adicionado isopropanol para solubilizar os cristais formados. Por fim, foi realizada a leitura da absorbância a $620 \mathrm{~nm}$ com auxílio de um leitor de placas (Anthos2020, Anthos Labtec Instruments, Wals, Áustria) $)^{35,36}$. Todos os experimentos foram repetidos de tratamento com US contínuo utilizando diferentes parâmetros dosimétricos

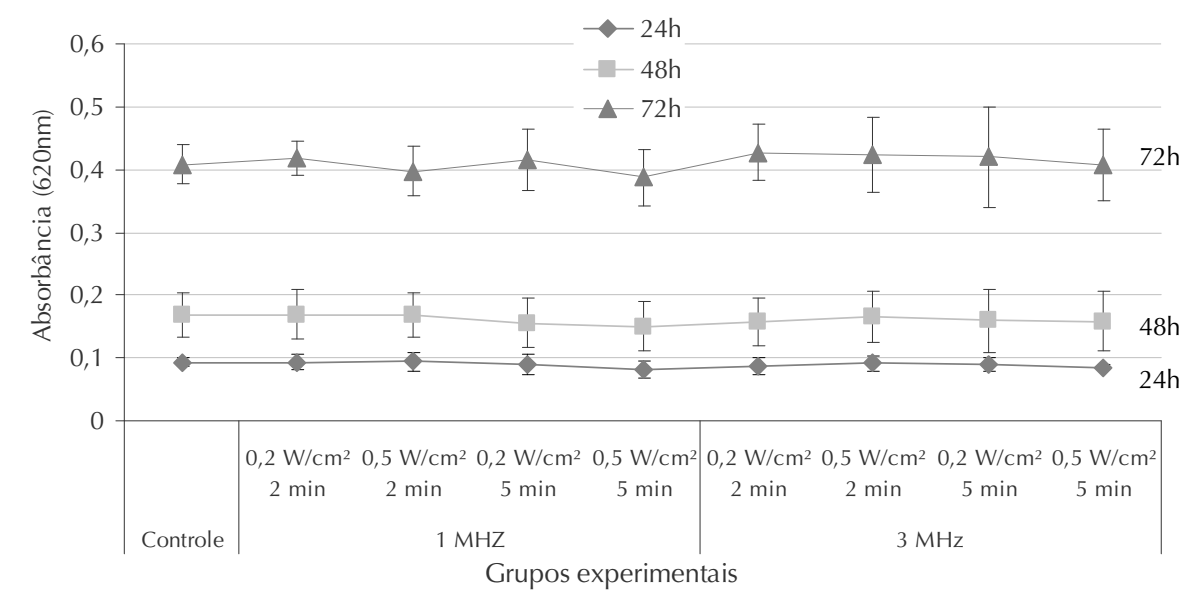

Figura 2 Proliferação e viabilidade de mioblastos na ausência (controle) e presença três vezes, de forma independente, e cada amostra foi analisada em quadruplicata.

\section{Análise estatística}

As comparações entre os grupos foram feitas utilizando análise de variância Anova. O teste de Dunnett foi utilizado para determinar diferenças significativas entre os grupos experimentais e o grupo controle. Valores de $p<0,05$ foram considerados estatisticamente significativos. Os dados foram analisados por meio do programa GraphPad Prism 4.0 (GraphPad Software, San Diego, CA, USA).

\section{RESULTADOS}

Quanto ao efeito do ultra-som contínuo sobre a viabilidade e proliferação celular, os resultados mostram que não houve diferença significativa na viabilidade e proliferação celular, avaliadas pelo método MTT, entre as culturas celulares tratadas com US (todos os parâmetros testados) e as culturas controle, após os diferentes períodos de incubação avaliados, conforme ilustrado na Figura 2. Além disso, foi possível verificar que houve um aumento do número de células com o aumento do período de incubação, tanto no grupo controle quanto nos tratados, conforme esperado (Figura 2). 


\section{DISCUSSÃO E CONCLUSÃO}

Segundo Johns ${ }^{19}$, a cultura celular é, por definição, um artefato; no entanto, permite que o investigador tenha um controle rigoroso sobre diversas variáveis do processo e possa fazer questionamentos de maneira mais sistemática. Portanto, estudos in vitro avaliando o potencial do US são importantes complementos dos estudos in vivo, e podem trazer maiores conhecimentos de forma a permitir a utilização desse recurso de forma mais eficaz e segura.

A linhagem celular C2C12 foi escoIhida neste experimento porque essas células são um subclone da linhagem de mioblastos $\mathrm{C} 2$, isoladas de células-satélites de ratos adultos ${ }^{37}$, que apresentam a maioria das características dos mioblastos normais e são comumente usadas como modelo para estudar a proliferação e a diferenciação de células musculares. O uso de linhagens celulares como modelos para a análise da proliferação celular elimina a possibilidade de o US interferir na produção de fatores de crescimento de células não-miogênicas contidas em culturas primárias, como fibroblastos e macrófagos ${ }^{31,38,39}$.

O presente estudo avaliou os efeitos do US contínuo em células musculares com o intuito de acumular maior conhecimento acerca da utilização desse recurso para o reparo tecidual após lesões, distrofias e outras condições nas quais a proliferação de células musculares é requerida. No entanto, os resultados evidenciam que o tratamento dessas células com o US não alterou sua proliferação, quando comparadas às células que não receberam tratamento.

É importante ressaltar que, apesar de o US não ter induzido aumento de proliferação, tampouco causou sua inibição, o que garante a utilização desse recurso no tratamento das lesões musculares com segurança.
Foi demonstrado que o US pulsado aumenta a proliferação de células satélites e de fibroblastos em modelo experimental de lesão contusa em músculo gastrocnêmio de rato40. Além disso, em outro estudo verificou-se que o tratamento com US melhora a extensibilidade muscular ${ }^{41}$, a produção de força após lesão por contração ${ }^{42}$ e pode aumentar a diferenciação das células musculares em modelo animal ${ }^{43}$. Visto que o US acelera o processo de reparo em diversos estudos in vivo, é possível que esse efeito dependa da ação coordenada de diferentes tipos celulares presentes em tecidos adjacentes ao tecido muscular, como por exemplo o conjuntivo. No presente estudo, pela escolha da utilização da linhagem celular, foi verificado somente o efeito do US sobre as células musculares. Assim, há a possibilidade de que o efeito do US no processo de reparo seja dependente da estimulação de outras células, que passariam a secretar fatores de crescimento envolvidos na proliferação e diferenciação das células musculares, em resposta a irradiação com US.

No entanto, outros autores que também utilizaram modelo experimental animal, foram igualmente incapazes de demonstrar aumento estatisticamente significativo na massa muscular ou na regeneração tecidual em lesões musculares tratadas com o US ${ }^{44-46}$. Essa controvérsia apóia a falta de evidência científica sobre a eficácia do US na reparação muscular $^{19,43,47}$.

Embora o US contínuo não tenha alterado a proliferação celular, o tratamento com esse recurso pode ter provocado uma modificação na síntese de fatores de crescimento e citocinas responsáveis pela diferenciação dessas células. Esta hipótese é respaldada pelo fato de que em experimentos in vivo o US aumentou a diferenciação das células musculares $^{40,42}$. Dessa forma, estudos futuros, utilizando técnicas adequadas, serão realizados na tentativa de responder essa questão.
Ikeda et al. ${ }^{48}$ avaliaram o efeito do US também sobre culturas de mioblastos C2C12, porém os parâmetros dosimétricos utilizados foram diferentes, dificultando a comparação com os presentes achados: foi utilizado o US pulsado e as células C2C12 foram cultivadas já em meio de diferenciação. Os autores verificaram que existe uma diferenciação das células musculares em osteoblastos e condroblastos em resposta ao tratamento com o US, nos parâmetros de $1,5 \mathrm{MHz}$ a uma intensidade de 70 $\mathrm{mW} / \mathrm{cm}^{2}$ por $20 \mathrm{~min}$.

Outros estudos in vitro demonstraram que os efeitos do US foram dependentes dos parâmetros utilizados e do tipo celular, sendo capaz de promover a proliferação de osteoblastos, fibroblas$\operatorname{tos}^{6,20,28,49}$, células endoteliais ${ }^{30}$ e condrócitos ${ }^{26}$. No entanto, outros autores não demonstraram de forma significativa os efeitos aditivos do US sobre a proliferação de fibroblastos ${ }^{50}$, de células provenientes de discos intervertebrais de bovinos ${ }^{26}$, de células uro-epiteliais ${ }^{29}$ ou de condrócitos ${ }^{51,52}$ ou até mesmo relatam diminuição no número de fibroblastos ${ }^{20}$ após o tratamento com US. Esse fato pode vir a reafirmar a possibilidade de que o US pode acelerar o processo de reparo muscular não diretamente pelo aumento na proliferação das células musculares e sim por estimular outros tipos celulares envolvidos no processo.

Com base no exposto, fica evidente que o enorme potencial terapêutico do US está longe de ser estabelecido em virtude de novas aplicações serem adicionadas regularmente ao seu repertório ${ }^{7}$. O presente estudo permitiu concluir que o US contínuo nos parâmetros utilizados não foi capaz de alterar a proliferação das células musculares, porém há necessidade da realização de estudos posteriores para o melhor entendimento de outros possíveis efeitos do US sobre essas células como, por exemplo, no processo de diferenciação, para o estabelecimento de protocolos adequados a serem utilizados para o reparo muscular e recuperação clínica dos pacientes. 


\section{REFERÊNCIAS}

1 Crisco JJ, Jokl P, Heinen GT, Connell MD, Panjabi MM. A muscle contusion injury model: biomechanics, physiology, and histology. Am J Sports Med. 1994;22(5):702-10.

2 Shi X, Garry DJ. Muscle stem cells in development, regeneration, and disease. Genes Dev. 2006;20:1692-708.

3 Kumar A, Mohan S, Newton J, Rehage M, Tran K, Baylink DJ. Pregnancy-associated plasma protein-A regulates myoblast proliferation and differentiation through an insulin-like growth factor-dependent mechanism. J Biol Chem. 2005;280(45):37782-9.

4 Dogra C, Hall SL, Wedhas N, Linkhart TA, Kumar A. Fibroblast growth factor inducible-14 (Fn14) is required for the expression of myogenic regulatory factors and differentiation of myoblasts into myotubes: evidence for Tweak-independent functions of Fn14 during myogenesis. J Biol Chem. 2007;282(20):15000-10.

5 Chargé SBP, Rudnicki MA. Cellular and molecular regulation of muscle regeneration. Physiol Rev. 2004;84:209-38.

6 Zhou S, Schmelz A, Seufferlein T, Li Y, Zhao J, Bachem MG. Molecular mechanisms of low- intensity pulsed ultrasound in human skin fibroblasts. J Biol Chem. 2004;279(52):54463-9.

7 Warden RKF, Kessler CK, Avin KG, Cardinal RE, Stewart RL. Ultrasound produced by a conventional therapeutic ultrasound unit accelerates fracture repair. Phys Ther. 2006;86(8):1118-27.

8 Hsieh YL. Effects of ultrasound and diclofenac phonophoresis on inflammatory painrelief: suppression of inducible nitric oxide synthase in arthritic rats. Phys Ther. 2006;86(1):39-49.

9 Azuma Y, Ito M, Harada Y, Takagi H, Ohta T, Jingushi S. Low-intensity pulsed ultrasound accelerates rat femoral fracture healing by acting on the various cellular reactions in the fracture callus. J Bone Miner Res. 2001;16:671-80.

10 Rubin C, Bolander M, Ryaby JP, Hadjiargyrou M. The use of low-intensity ultrasound to accelerate the healing of fractures. J Bone Joint Surg. 2001;83(A):259-70.

11 Franco AD, Pereira LE, Groschitz M, Aimbire F, Martins RABL, Carvalho RA. Análise do efeito do ultra-som no edema inflamatório agudo: estudo experimental. Fisioter Mov. 2005;18(2):19-24.

12 Mendonça AC, Ferreira AS, Barbieri $\mathrm{CH}$, Thomazine JA, Mazzer N. Efeitos do ultra-som pulsado de baixa intensidade sobre a cicatrização por segunda intenção de lesões cutâneas totais em ratos. Acta Ortop Bras. 2006;14(3):152-7.

13 Ferrari RJ, Picchi LD, Botelho AP, Minamoto V. Processo de regeneração na lesão muscular: uma revisão. Fisioter Mov. 2005;18(2):63-71.
14 Speed CA. Therapeutic ultrasound in soft tissue lesions. Rheumatology (Oxford). 2001;40:1331-6.

15 Mitragotri S. Healing sound: the use of ultrasound in drug delivery and other therapeutic applications. Nat Rev Drug Discov. 2005;4:255-60.

16 Sundaram J, Mellein BR, Mitragotri S. An experimental and theoretical analysis of ultrasound-induced permeabilization of cell membranes. Biophys J. 2003;84(5):3087-101.

17 Baker KG, Robertson VJ, Duck FA. A review of therapeutic ultrasound: biophysical effects. Phys Ther. 2001;81(7):1351-8.

18 Blume K, Matsuo E, Lopes MS, Lopes LG. Dosimetria proposta para o tratamento por ultra-som: uma revisão de literatura. Fisioter Mov. 2005;18(3):55-64.

19 Johns LD. Nonthermal effects of therapeutic ultrasound: the frequency resonance hypothesis. J Athl Train. 2002;37(3):293-9.

20 De Deyne PG, Kirsch-Volders M. In vitro effects of therapeutic ultrasound on the nucleus of human fibroblast. Phys Ther. 1995;75:629-34.

21 Doan N, Reher P, Meghji S, Harris M. In vitro effects of therapeutic ultrasound on cell proliferation, protein synthesis and cytokine production by human fibroblasts, osteoblasts and monocytes. J Oral Maxillofac Surg. 1999;57:409-19.

22 Reher P, Doan N, Bradnock B, Meghji S, Harris M. Effect of ultrasound on the productin of IL-8, basic FGF and VEGF. Cytokine. 1999;11:416-23.

23 Maddi A, Hai H, Ong ST, Sharp L, Harris M, Meghji S. Long-wave ultrasound may enhance bone regeneration by altering OPG/RANKL ratio in human osteoblast-like cells. Bone. 2006;39:283-8.

24 Tang CH, Yang RS, Huang TH, Lu DY, Chuang WJ, Huang TF, et al. Ultrasound stimulates cyclooxygenase-2 expression and increases bone formation through integrin, focal adhesion kinase, phosphatidylinositol 3-kinase, and akt pathway in osteoblasts. Mol Pharmacol. 2006;69(6):2047-57.

25 Park K, Hoffmeister B, Han DK, Hasty K. Therapeutic ultrasound effects on interleukin-1B stimulated cartilage construct in vitro. Ultrasound Med Biol. 2007;33(2):286-95.

26 Miyamoto K, An HS, Sah RL, Akeda K, Okuma M, Otten $\mathrm{L}$, et al. Exposure to pulsed low-intensity ultrasound stimulates extracellular matrix metabolism of bovine intervertebral disc cells cultured in alginate beads. Spine. 2005;30(21):2398-405.

27 Mukai S, Ito H, Nakagawa Y, Akiyama H, Miyamoto M, Nakamura T. Transforming growth factor B1 mediates the effects of low-intensity pulsed ultrasound in chondrocytes. Ultrasound Med Biol. 2005;31(12):1713-21. 


\section{Referências (cont.)}

28 Lai J, Pittelkow MR. Physiological effects of ultrasound mist on fibroblast. Int J Dermatol. 2007;46(6):587-93.

29 Hill GE, Fenwick S, Matthews BJ, Chivers RA, Southgate J. The effect of low-intensity pulsed ultrasound on repair of epithelial cell monolayers in vitro. Ultrasound Med Biol. 2005;31(12):1701-6.

30 Mizrahi N, Seliktar D, Kimmel E. Ultrasound-induced angiogenic response in endothelial cells. Ultrasound Med Biol. 2007;33(11):1818-29.

31 Lee MH, Jang MH, Kim EK, Han SW, Cho SY. Nitric oxide induces apoptosis in mouse C2C12 myoblast cells. J Pharmacol Sci. 2005;97:369-76.

32 Amaral AC. Influência da terapia a laser de baixa intencidade em células precursoras miogênicas (in vitro) e durante a regeneração muscular (in vivo) [dissertação] São Carlos: Universidade Federal de São Carlos; 2004.

33 Amack JD, Mahadevan MS. The myotonic dystrophy expanded CUG repeat tract is necessary but not sufficient to disrupt C2C12 myoblast differentiation. Hum Mol Genet. 2001;10(8):1879-87.

34 Löster K, Horstkorte R. Enzymatic quantification of cellmatrix and cell-cell adhesion. Micron. 2000;31(1):41-53.

35 Mosmann T. Rapid colorimetric assay for cellular growth and survival: application to proliferation and cytotoxicity assays. J Immunol Methods. 1983;65(2):55-63.

36 Woerdenbag HJ, Merfort I, Passreiter CM, Schmidt TJ, Willuhn G, Van Uden W, et al. Cytotoxicity of flavonoids and sesquiterpene lactones from Arnica species against the GLC4 and the COLO 320 cell lines. Planta Med. 60(5):434-7.

37 Yaffe D, Saxel D. Serial passaging and differentiation of myogenic cells isolated from dystrophic mouse muscle. Nature. 1977;270:725-7.

38 Chandran R, Knobloch TJ, Anghelina M, Agarwal S. Biomechanical signals upregulate myogenic gene induction in the presence or absence of inflammation. Am J Physiol Cell Physiol. 2007;293:C267-76.

39 Ferreira MPP, Ferrari RAM, Gravalos ED, Martins MD, Bussadori SK, Gonzales DAB, et al. Effect of low-energy GaAlAs and InGaAIP laser irradiation on the viability of C2C12 myoblasts in a muscle injury model. Photomed Laser Surg. 2009;27(6):901-6.

40 Rantanen J, Thorsson O, Wollmer P, Hurme T, Kalimo H. Effects of therapeutic ultrasound on the regeneration of skeletal myofibers after experimental muscle injury. Am J Sports Med. 1999;27(1):54-9.
41 Wessling KC, DeVane DA, Hylton CR. Effects of static stretch versus static stretch and ultrasound combined on triceps surae muscle extensibility in healthy women. Phys Ther. 1987;67:674-9.

42 Karnes JL, Burton HW. Continuous therapeutic ultrasound accelerates repair of contraction-induced skeletal muscle damage in rats. Arch Phys Med Rehabil. 2002;83:1-4.

43 Piedade MCB, Galhardo MS, Battlehner CN, Ferreira MA, Caldini EG, Toledo OMS. Effect of ultrasound therapy on the repair of gastrocnemius muscle injury in rats. Ultrasonics. 2008;48:403-11.

44 Wilkin LD, Merrick MA, Kirby TE, Devor ST. Influence of therapeutic ultrasound on skeletal muscle regeneration following blunt contusion. Int J Sports Med. 2004;25:73-7.

45 McBrier NM, Lekan JM, Druhan LJ, Devor ST, Merrick MA. Therapeutic ultrasound decreases mechano-growth factor messenger ribonucleic acid expression after muscle contusion injury. Arch Phys Med Rehabil. 2007;88:936-40.

46 Markert CD, Merrick MA, Kirby TE, Devor ST. Nonthermal ultrasound and exercise in skeletal muscle regeneration. Arch Phys Med Rehabil. 2005;86:1304-10.

47 Järvinen TA, Järvinen TLN, Järvinen $M$, Kääriäinen $M$, Kalimo H, Järvinen M. Muscle injuries: biology and treatment. Am J Sports Med. 2005;33;745-64.

48 Ikeda K, Takayama T, Suzuki N, Shimada K, Otsuka K, Ito K. Effects of low-intensity pulsed ultrasound on the differentiation of C2C12 cells. Life Sci. 2006;79(20):1936-43.

49 Chen YJ, Wang CJ, Yang KD, Chang PR, Huang HC, Huang YT, et al. Pertussis toxin-sensitive GKi protein and ERK-dependent pathways mediate ultrasound promotion of osteogenic transcription in human osteoblasts. FEBS Lett. 2003;554(1):154-8.

50 Oliveira RF, Oliveira DA, Monteiro W, Zangaro RA, Magini M, Soares CP. Comparison between the effect of low-level laser therapy and low-intensity pulsed ultrasonic irradiation in vitro. Photomed Laser Surg. 2008;26(1):6-9.

51 Parvizi J, Wu CC, Lewallen DG, Greenleaf JF, Bolander ME. Low-intensity ultrasound stimulates proteoglycan synthesis in rat chondrocytes by increasing aggrecan gene expression. J Orthop Res. 1999;17(4):488-94.

52 Zhang ZJ, Huckle J, Francomano CA, Spencer RGS. The effects of pulsed low-intensity ultrasound on chondrocyte viability, proliferation, gene expression and matrix production. Ultrasound Med Biol. 2003;29(11):1645-51. 